\title{
High Apolipoprotein E Levels Predict Adverse Limb Events in Patients with Peripheral Artery Disease Due to Peripheral Artery Disease Undergoing Endovascular Treatment and On-Statin Treatment
}

\author{
Tatsuya Fukase, ${ }^{1}$ MD, Tomotaka Dohi, ${ }^{1}$ MD, Yoshiteru Kato, ${ }^{1}$ MD, Yuichi Chikata, ${ }^{1}$ MD, \\ Norihito Takahashi, ${ }^{1}$ MD, Hirohisa Endo, ${ }^{1}$ MD, Shinichiro Doi, ${ }^{1}$ MD, Hiroki Nishiyama, ${ }^{1}$ MD, \\ Iwao Okai, ${ }^{1}$ MD, Hiroshi Iwata, ${ }^{1}$ MD, Shinya Okazaki, ${ }^{1}$ MD, Kikuo Isoda, ${ }^{1}$ MD, \\ Katsumi Miyauchi, ${ }^{1}$ MD, Hiroyuki Daida, ${ }^{1}$ MD and Tohru Minamino, ${ }^{1,2}$ MD
}

\begin{abstract}
Summary
Little is known about the association between limb prognosis in peripheral artery disease and apolipoprotein $\mathrm{E}$ (apoE). We evaluated the long-term impact of apoE on adverse limb events in patients with intermittent claudication receiving statin treatment.

A total of 218 consecutive patients (mean age, $73 \pm 8$ years; $81 \%$ men) with intermittent claudication who underwent their first intervention between 2009 and 2020 were included in this study. All patients had achieved LDL-C $<100 \mathrm{mg} / \mathrm{dL}$ on statin treatment and were divided into two groups based on the apoE value $(\geq 4.7$ or $<4.7 \mathrm{mg} / \mathrm{dL}$ ). We evaluated the incidence of major adverse limb events (MALEs), including vessel revascularization and limb ischemia development.

A total of 39 and 179 patients were allocated to the higher and lower apoE groups, respectively. Compared to the lower apoE group, the higher apoE group had a significantly higher total cholesterol level, triglyceride level, and non-high-density lipoprotein cholesterol level. During the median follow-up period of 3.6 years, 30 patients $(13.8 \%)$ developed MALEs. Kaplan-Meier analysis revealed that the cumulative incidence of MALEs in the higher apoE group was significantly higher than that in the lower apoE group $(44.0 \%$ versus $21.6 \%$, $\log$ rank test, $P=0.002)$. During multivariable Cox hazard analysis, higher apoE level $(\geq 4.7 \mathrm{mg} / \mathrm{dL}$ ) (hazard ratio, 2.61; $95 \%$ confidence interval, $1.18-5.70, P=0.019)$ was the only strong independent predictor of MALEs.

ApoE levels could be a strong predictor and residual risk for long-term limb prognosis in patients with intermittent claudication and achieving LDL-C $<100 \mathrm{mg} / \mathrm{dL}$ with statin treatment.
\end{abstract}

(Int Heart J 2021; 62: 872-878)

Key words: Major adverse limb events, Target vessel revascularization, Intermittent claudication, Low-density lipoprotein cholesterol, Lipid profiles

$\mathrm{P}$ atients with intermittent claudication due to peripheral artery disease (PAD) experience a slow disease progression; however, the frequency of revascularization, development of critical limb ischemia, and the risk of amputation increases without proper prevention and treatment. In addition, intermittent claudication as a manifestation of PAD is a strong marker for high morbidity and mortality due to generalized atherosclerosis, including cardiovascular and cerebrovascular diseases. Thus, symptoms and quality of life in patients with PAD have to improve and the risk of cardiovascular and limb events, such as supervised exercise training, smoking cessation, blood pressure management, lipid-lowering, glycemic control, antiplatelet therapy, and anticoagulants therapy such as direct oral anticoagulants should be reduced. ${ }^{1-7)}$ Many studies focusing on lipid-lowering therapies have reported that lowering low-density lipoprotein cholesterol (LDL-C) in patients with intermittent claudication prevents cardiovascular morbidity and mortality ${ }^{8,9)}$ and improves claudication symptoms and exercise tolerance. ${ }^{10)}$ The American College of Cardiology/American Heart Association guideline provides a class I indication for statin treatment in all patients with intermittent claudication due to PAD. ${ }^{11}$ However, few studies have examined the effectiveness of lowering LDL-C in preventing ischemic limb events compared to cardiovascular events. In addition, little is known about the association between risk of adverse limb events and lipid profile (except for LDL-C); particularly, nothing

From the ${ }^{1}$ Department of Cardiovascular Biology and Medicine, Juntendo University Graduate School of Medicine, Tokyo, Japan and ${ }^{2}$ Japan Agency for Medical Research and Development-Core Research for Evolutionary Medical Science and Technology (AMED-CREST), Tokyo, Japan.

Address for correspondence: Tomotaka Dohi, MD, Department of Cardiovascular Biology and Medicine, Juntendo University Graduate School of Medicine,

2-1-1 Hongo, Bunkyo-ku, Tokyo 113-0033, Japan. E-mail: tdohi@juntendo.ac.jp

Received for publication December 24, 2020. Revised and accepted March 2, 2021.

Released in advance online on J-STAGE July 17, 2021.

doi: $10.1536 /$ ihj.20-816

All rights reserved by the International Heart Journal Association. 
is known about the role of apoE in the incidence of adverse limb events. In spite of the fact that apoE strongly contributes to arteriosclerosis including cardiovascular disease, ${ }^{12)}$ the related report is only between the apoE genotype and prevalence of PAD. ${ }^{13)}$ Thus, we aimed to evaluate the long-term impact of apoE on adverse limb events in patients with intermittent claudication due to PAD who underwent endovascular treatment and achieved lowering LDL-C on-statin treatment.

\section{Methods}

Study population: This single-center, observational, retrospective cohort study was conducted at our institution. We enrolled 400 consecutive patients with intermittent claudication due to PAD who underwent their first intervention between January 2009 and March 2020. In patients with an appropriate history and physical examination, we diagnosed PAD when the ankle-brachial index, which is defined as the ratio of the systolic blood pressure measured over the ankle to the systolic blood pressure measured over the brachial artery, was $\leq 0.9 .^{14)}$ In addition, we used duplex ultrasonography as needed to identify the location and severity of arterial obstruction, which has $85 \%-90 \%$ sensitivity and $>95 \%$ specificity for detecting stenosis $>50 \% .^{15}$ Advanced vascular imaging techniques, such as computed tomographic angiography and magnetic resonance angiography, were reserved for patients whose diagnoses remained uncertain following non-invasive testing or in whom intervention was anticipated. ${ }^{16,17)}$ The choice of treatment was based on a multidisciplinary evaluation, as mentioned above. Generally, patients with intermittent claudication were initially treated using pharmacotherapy (some antihypertensive drugs, statins, oral hypoglycemic agents, insulin, antiplatelet agents, etc.), exercise therapy (supervised exercise training, or if it is not feasible unsupervised exercise training), and smoking cessation guidance. However, if these conservative therapies were ineffective, patients were eligible for intervention. The exclusion criteria were as follows: 1) patients not taking statins at the time of the first intervention, 2) patients not achieving the lowering of LDL-C (with a goal of $<100 \mathrm{mg}$ / $\mathrm{dL}),{ }^{18)}$ 3) patients undergoing hemodialysis, and 4) patients with missing data on the lipid profile. Finally, 218 patients were divided into two groups (higher apoE and lower apoE) according to the preprocedural apoE levels based on the optimal cutoff value of apoE using receiver operating characteristic curve (ROC) analysis.

This study was approved by the ethics committee of our institution and was performed following the Declaration of Helsinki. All participants provided written informed consent.

Data collection and definitions: Data on patient characteristics were collected from the institutional database. Blood samples were collected early in the morning after an overnight fast. Baseline plasma samples from the patients were thawed and assayed for each plasma parameter using commercially available analytic systems. Regarding lipid profile, total cholesterol, high-density lipoprotein cholesterol, direct LDL-C, and triglyceride levels were assayed using a LABOSPECT 008 $\alpha$ (Hitachi, Ltd., Tokyo,
Japan) at our institution. The measurement of the following samples was outsourced to an external specialist; lipoprotein (a) was assayed using a latex-enhanced immunonephelometric assay, and apoA-I, apoB, and apoE were simultaneously measured on the same device using an immunoassay. Patients with a blood pressure $>140 / 90$ $\mathrm{mmHg}$ or those receiving antihypertensive drugs were considered hypertensive. Diabetes mellitus was defined as either a hemoglobin A1c level $\geq 6.5 \%$ or the administration of oral hypoglycemic drugs or insulin injections. Chronic kidney disease was defined as an estimated glomerular filtration rate $<60 \mathrm{~mL} /$ minute $/ 1.73 \mathrm{~m}^{2}$, as calculated by the Diet in Renal Disease equation modified with a Japanese coefficient using the baseline serum creatinine. ${ }^{19)}$

Study endpoint: The endpoint of this study was major adverse limb events (MALEs), defined as a composite of clinically driven target vessel revascularization and development of limb ischemia. Clinically driven target vessel revascularization was defined as re-intervention at the target vessel when symptoms occurred, and significant stenosis was detected by duplex ultrasonography or when the ankle-brachial index value markedly decreased. In addition, we evaluated the development of limb ischemia when we detected newly developed ischemic rest pain and tissue loss.

Clinical follow-up data were collected from the patients' medical records or by contacting the patients or their families if they had not been followed up in our institution after the intervention. Information about the circumstances and date of death was obtained from the families of patients who died at home, and details of events associated with the cause of death were supplied by staff of other hospitals or clinics in which the patient had been admitted. Investigators blinded to the study collected all the data.

Statistical analysis: Categorical data were presented as numbers and percentages and compared using the chisquared test. Continuous variables were expressed as mean \pm standard deviation or as median and interquartile range and were compared using one-way analysis of variance or the Kruskal-Wallis test. Univariate analysis of the relationship between the apoE level and variables was performed using Pearson's correlation analysis. A KaplanMeier analysis for the cumulative incidence of MALEs was used to compare the two groups based on the cutoff value of apoE using ROC analysis, and differences between the groups were assessed using the log-rank test. The multivariable Cox model was performed by stepwise selection with entry/stay criteria of $0.20 / 0.20$. Regarding MALEs, apoE, total cholesterol, triglyceride, non-highdensity lipoprotein cholesterol, and pre-treatment anklebrachial index were included in the list of candidate covariates.

All probabilities were expressed as two-tailed values, with statistical significance inferred at $P<0.05$. All confidence intervals (CIs) were computed at the $95 \%$ level. All data were analyzed using JMP version 12.0 for Macintosh (SAS Institute, Cary, NC, USA). 


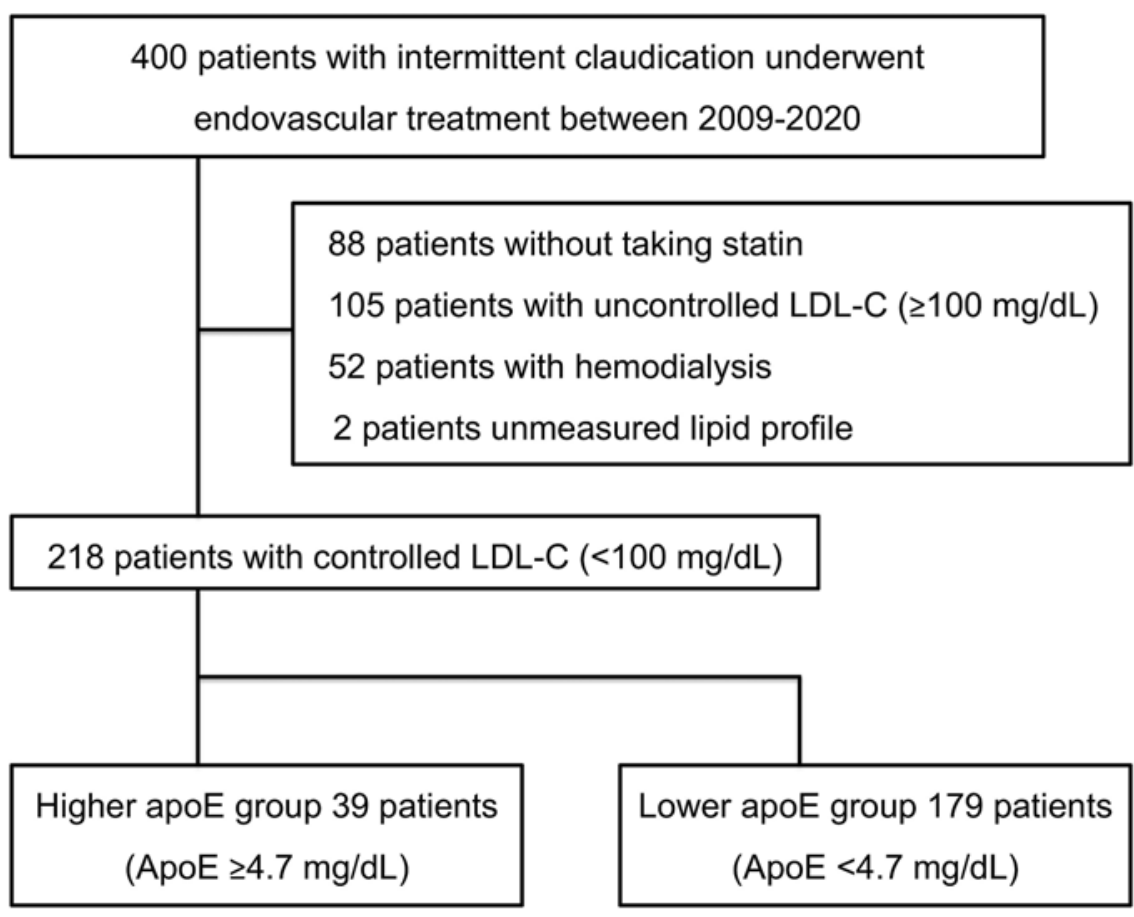

Figure 1. Study flow chart. Among 400 patients, we excluded the following persons: patients not taking statin, with uncontrolled LDL-C, on hemodialysis, and with an unmeasured lipid profile. We studied 218 patients with controlled LDL-C on statin. The patients were divided into two groups according to the preprocedural apoE levels based on the optimal cutoff value of apoE using ROC analysis (dichotomized to $\geq 4.7$ or $<4.7 \mathrm{mg} / \mathrm{dL}$ ). ApoE indicates apolipoprotein E; and LDL-C, low-density lipoprotein cholesterol.

\section{Results}

Baseline characteristics: Overall, we studied 218 patients on statins with LDL-C $<100 \mathrm{mg} / \mathrm{dL}$. (Figure 1). Their baseline clinical characteristics are summarized in Table I. The mean age was $73 \pm 8$ years, and $81 \%$ of the patients were men. The prevalence of hypertension, diabetes mellitus, and smoking was $84 \%, 56 \%$, and $88 \%$, respectively. Although the prevalence of patients with chronic kidney disease was $37 \%$, the mean estimated glomerular filtration rate of $66.8 \mathrm{~mL} /$ minute $/ 1.73 \mathrm{~m}^{2}$ was within normal limits. All patients took statins, but the use of any other lipidlowering agents, including ezetimibe, fibrates, and eicosapentaenoic acid, was less than $10 \%$.

Relationships between $\mathbf{a p o E}$ and risk factors for MALEs: Overall, median and mean apoE levels were 3.6 $\mathrm{mg} / \mathrm{dL}$ (interquartile range: $3.0,4.3$ ) and $3.8 \mathrm{mg} / \mathrm{dL}$ (standard deviation: 0.6), respectively. When we analyzed the ROC curves of apoE to predict future MALEs, the area under the curve of ROC for apoE levels was 0.64, with the highest discriminating sensitivity and specificity of 0.53 and 0.87 , respectively, at apoE of $4.7 \mathrm{mg} / \mathrm{dL}$ (Supplemental Figure). Thirty-nine patients (18\%) were allocated to the higher-apoE group (apoE level $\geq 4.7 \mathrm{mg} / \mathrm{dL}$ ), whereas 179 patients $(82 \%)$ were included in the lowerapoE group (apoE level $<4.7 \mathrm{mg} / \mathrm{dL}$ ) (Table I). No significant differences were seen in age, sex, hypertension, diabetes mellitus, smoking, chronic kidney disease, or any oral medication between the higher and lower apoE groups (all $P \geq 0.05$ ). Compared to the lower-apoE group, the higher-apoE group had significantly higher total cholesterol, triglyceride, non-high-density lipoprotein cholesterol, apoA-I, apoB, and apoE levels (all $P<0.05$ ). We summarized the univariate analysis of the relationship between apoE levels and risk factors for MALEs in Table II. As mentioned above, there were significant relationships between apoE and total cholesterol levels, triglyceride levels, and non-high-density lipoprotein cholesterol levels (all $P<0.001)$. By contrast, apoE levels did not correlate with LDL-C levels.

ApoE as a predictive factor for MALEs: The median follow-up duration was 3.6 years (interquartile range, 1.26.2 years), and the prognostic data were fully documented during the entire follow-up period. During the follow-up, 30 patients (13.8\%) developed MALEs, including clinically driven target vessel revascularization in 30 and development of limb ischemia in 4. The Kaplan-Meier analysis indicated that the cumulative incidence of MALEs in the higher-apoE group was significantly higher than that in the lower-apoE group (44.0\% versus $21.6 \%$, log-rank test, $P=0.002$ ) (Figure 2). Furthermore, Table III presents results of Cox proportional hazard regression analyses for MALEs. Higher apoE level $(\geq 4.7 \mathrm{mg} / \mathrm{dL}$ as the cutoff value), total cholesterol (10 mg increase), triglyceride (10 mg increase), non-high-density lipoprotein cholesterol (10 mg increase), and lower ankle-brachial in$\operatorname{dex}(<0.68$ as the median value) were applicable as covariates. Multivariable Cox hazard analysis revealed that a 
Table I. Patients' Baseline Clinical Characteristics

\begin{tabular}{|c|c|c|c|c|}
\hline Clinical characteristics & $\begin{array}{c}\text { Overall } \\
\text { (218 patients) }\end{array}$ & $\begin{array}{l}\text { Higher apoE group } \\
\text { (39 patients) }\end{array}$ & $\begin{array}{l}\text { Lower apoE group } \\
\text { (179 patients) }\end{array}$ & $P$-value \\
\hline Age, years & $73 \pm 8$ & $72 \pm 9$ & $73 \pm 7$ & 0.279 \\
\hline Male sex, $n(\%)$ & $177(81)$ & $27(69)$ & $147(82)$ & 0.081 \\
\hline BMI, $\mathrm{kg} / \mathrm{m}^{2}$ & $23.8 \pm 3.1$ & $23.9 \pm 3.1$ & $22.8 \pm 3.1$ & 0.141 \\
\hline $\mathrm{SBP}, \mathrm{mmHg}$ & $142.7 \pm 23.4$ & $143.1 \pm 20.3$ & $142.6 \pm 24.0$ & 0.901 \\
\hline $\mathrm{DBP}, \mathrm{mmHg}$ & $73.2 \pm 14.2$ & $73.4 \pm 15.5$ & $73.2 \pm 13.9$ & 0.943 \\
\hline TC level, mg/dL & $149 \pm 20$ & $161 \pm 20$ & $146 \pm 21$ & $<0.001$ \\
\hline TG level, mg/dL & $119 \pm 54$ & $170 \pm 92$ & $109 \pm 42$ & $<0.001$ \\
\hline HDL-C level, mg/dL & $46 \pm 13$ & $47 \pm 16$ & $46 \pm 13$ & 0.466 \\
\hline Non HDL-C level, mg/dL & $103 \pm 18$ & $113 \pm 21$ & $100 \pm 17$ & $<0.001$ \\
\hline LDL-C level, mg/dL & $76 \pm 14$ & $76 \pm 14$ & $77 \pm 15$ & 0.656 \\
\hline Lp (a) level, mg/dL & $23(11,42)$ & $19(9,38)$ & $24(11,44)$ & 0.710 \\
\hline ApoA-I level, mg/dL & $129 \pm 26$ & $139 \pm 29$ & $127 \pm 25$ & 0.013 \\
\hline ApoB level, mg/dL & $73 \pm 12$ & $82 \pm 14$ & $71 \pm 12$ & $<0.001$ \\
\hline ApoE level, mg/dL & $3.8 \pm 0.6$ & $5.3 \pm 0.8$ & $3.4 \pm 0.6$ & $<0.001$ \\
\hline eGFR, $\mathrm{mL} / \mathrm{minute} / 1.73 \mathrm{~m}^{2}$ & $66.8 \pm 19.8$ & $69.9 \pm 18.1$ & $66.2 \pm 20.1$ & 0.289 \\
\hline HbA1c level, \% & $6.5 \pm 1.0$ & $6.7 \pm 1.1$ & $6.5 \pm 0.9$ & 0.243 \\
\hline Hs-CRP level, g/dL & $0.1(0.04,0.23)$ & $0.1(0.04,0.21)$ & $0.1(0.04,0.24)$ & 0.888 \\
\hline NT-proBNP level, pg/mL & $154.2(78.5,526.3)$ & $135.5(68.0,452.8)$ & $155.9(80.9,545.0)$ & 0.292 \\
\hline Pre-treatment ABI & $0.68 \pm 0.15$ & $0.69 \pm 0.13$ & $0.68 \pm 0.16$ & 0.857 \\
\hline Post-treatment ABI & $0.94 \pm 0.16$ & $0.95 \pm 0.16$ & $0.93 \pm 0.16$ & 0.559 \\
\hline \multicolumn{5}{|l|}{ Comorbidity } \\
\hline Hypertension, $n(\%)$ & $184(84)$ & $36(92)$ & $148(83)$ & 0.107 \\
\hline Diabetes mellitus, $n(\%)$ & $122(56)$ & $26(67)$ & $96(54)$ & 0.133 \\
\hline Chronic kidney disease, $n(\%)$ & $81(37)$ & $11(28)$ & $70(39)$ & 0.195 \\
\hline Smoking, $n(\%)$ & $192(88)$ & $34(87)$ & $158(88)$ & 0.851 \\
\hline Coronary artery disease, $n(\%)$ & $113(52)$ & $18(46)$ & $95(53)$ & 0.433 \\
\hline Cerebrovascular disease, $n(\%)$ & $40(17)$ & $5(13)$ & $35(20)$ & 0.308 \\
\hline Congestive heart failure, $n(\%)$ & $10(5)$ & $3(8)$ & $7(4)$ & 0.339 \\
\hline \multicolumn{5}{|l|}{ Medication } \\
\hline Statin, $n(\%)$ & $218(100)$ & $39(100)$ & $179(100)$ & \\
\hline Ezetimibe, $n(\%)$ & $22(10)$ & $3(8)$ & $19(11)$ & 0.571 \\
\hline Fibrates, $n(\%)$ & $7(3)$ & $2(5)$ & $5(3)$ & 0.481 \\
\hline $\mathrm{EPA}, n(\%)$ & $17(8)$ & $2(5)$ & $15(8)$ & 0.472 \\
\hline Aspirin, $n(\%)$ & $177(81)$ & $33(85)$ & $144(80)$ & 0.538 \\
\hline P2Y12 receptor inhibitor, $n(\%)$ & $153(70)$ & $24(62)$ & $129(72)$ & 0.201 \\
\hline Cilostazol, $n(\%)$ & $77(35)$ & $12(31)$ & $65(36)$ & 0.508 \\
\hline Prostanoids, $n(\%)$ & $33(15)$ & $7(18)$ & $26(15)$ & 0.605 \\
\hline Anticoagulants & $23(11)$ & $4(10)$ & $19(11)$ & 0.947 \\
\hline$\beta$-blocker, $n(\%)$ & $73(33)$ & $9(23)$ & $64(36)$ & 0.119 \\
\hline $\mathrm{CCB}, n(\%)$ & $120(55)$ & $22(56)$ & $98(55)$ & 0.850 \\
\hline ACE-i/ARB, $n(\%)$ & $124(57)$ & $20(51)$ & $104(58)$ & 0.437 \\
\hline OHA, $n(\%)$ & $86(39)$ & $20(51)$ & $66(37)$ & 0.098 \\
\hline Insulin, $n(\%)$ & $26(12)$ & $7(18)$ & $19(11)$ & 0.222 \\
\hline
\end{tabular}

Higher apoE group, patients with an apoE level $\geq 4.7 \mathrm{mg} / \mathrm{dL}(n=39)$. ABI indicates ankle-brachial index; ACE-i, angiotensin-converting enzyme inhibitor; ApoA-I, apolipoprotein A-I; ApoB, apolipoprotein B; ApoE, apolipoprotein $\mathrm{E}$; ARB, angiotensin receptor blocker; BMI, body mass index; $\mathrm{CCB}$, calcium channel blocker; DBP, diastolic blood pressure; eGFR, estimated glomerular filtration rate; EPA, Eicosapentaenoic acid; HbA1c, Hemoglobin A1c; HDL-C, high-density lipoprotein cholesterol; Hs-CRP, high-sensitivity C-reactive protein; LDL-C, low-density lipoprotein cholesterol; Lp (a), lipoprotein (a); NT-proBNP, N-terminal pro-brain natriuretic peptide; OHA, oral hypoglycemic agent; SBP, systolic blood pressure; TC, total cholesterol; and TG, triglyceride.

high apoE level ( $\geq 4.7 \mathrm{mg} / \mathrm{dL}$ ) (hazard ratio [HR], 2.61; $95 \% \mathrm{CI}, 1.18-5.70, P=0.019)$ was the only strong independent predictor of MALEs. Furthermore, continuous variables of the apoE levels were also significantly related to the incidence of MALEs (per log apoE 1 increase, HR, 4.01; 95\% CI, 1.00-15.0, $P=0.049)$.

\section{Discussion}

The major findings of this study are as follows: (1) Higher apoE levels were significantly associated with a higher incidence of MALEs compared to lower apoE levels in patients on statin with LDL-C $<100 \mathrm{mg} / \mathrm{dL}$; (2) in the multivariable Cox hazard analysis, a high apoE level was the only strong independent predictor of MALEs; and (3) continuous variables of the apoE levels (per log apoE 
1 increase) were also significantly related to the incidence of MALEs.

Although the association between apoE alleles and

Table II. Univariate Analysis of the Relationship Between ApoE Level and Variables

\begin{tabular}{lrr}
\hline \multicolumn{1}{c}{ Variables } & \multicolumn{1}{c}{$r$} & $P$-value \\
\hline Age, years & -0.09 & 0.169 \\
BMI, kg/m² & 0.21 & 0.002 \\
SBP, mmHg & 0.09 & 0.199 \\
DBP, mmHg & 0.09 & 0.198 \\
TC level, mg/dL & 0.44 & $<0.001$ \\
TG level, mg/dL & 0.42 & $<0.001$ \\
HDL-C level, mg/dL & 0.10 & 0.132 \\
Non HDL-C level, mg/dL & 0.42 & $<0.001$ \\
LDL-C level, mg/dL & 0.11 & 0.095 \\
Lp (a) level, mg/dL & 0.04 & 0.570 \\
ApoA-I level, mg/dL & 0.26 & $<0.001$ \\
ApoB level, mg/dL & 0.43 & $<0.001$ \\
eGFR, mL/minute/1.73 m ${ }^{2}$ & 0.01 & 0.831 \\
HbAlc level, \% & 0.08 & 0.231 \\
Hs-CRP level, g/dL & 0.01 & 0.861 \\
NT-proBNP level, pg/mL & -0.04 & 0.597 \\
\hline
\end{tabular}

ApoA-I indicates apolipoprotein A-I; ApoB, apolipoprotein B; ApoE, apolipoprotein E; BMI, body mass index; DBP, diastolic blood pressure; eGFR, estimated glomerular filtration rate; HbA1c, Hemoglobin A1c; HDL-C, high-density lipoprotein-cholesterol; Hs-CRP, high-sensitivity C-reactive protein; LDL-C, low-density lipoprotein-cholesterol; Lp (a), lipoprotein (a); NT-proBNP, N-terminal pro-brain natriuretic peptide; SBP, systolic blood pressure; TC, total cholesterol; and TG, triglyceride.
Alzheimer's disease has been reported in recent years, ${ }^{20)}$ it is strongly implicated even in cardiovascular disease. ${ }^{12)}$ In addition, there is some discussion about the relationship between the apoE genotype and prevalence of PAD ${ }^{13)}$ but the correlation between plasma apoE levels and the incidence of adverse events in patients with PAD has never been reported in real clinical practice. Therefore, this may be the first study to demonstrate clinical significance of systemic blood apoE levels in PAD patients with intermittent claudication during long-term follow-up.

ApoE was discovered as a component of very lowdensity lipoprotein in the $1970 \mathrm{~s}^{21,22)}$ ApoE is one of the major apolipoproteins present as part of several lipoprotein particle classes, including chylomicron remnants, very low-density lipoprotein, intermediate-density lipoprotein, and some high-density lipoprotein; it is a major ligand for LDL receptors and plays a role in cholesterol metabolism. ${ }^{23)}$ ApoE has structural features of the three isoforms (apoE2, apoE3, and apoE4), of which apoE2 and apoE4 increase the risk for cardiovascular diseases. Some studies have demonstrated that apoE2 is associated with increased plasma triglyceride levels. This mechanism of onset has been ascribed to defective LDL receptor binding of apoE2, which impairs the clearance of triglyceride-rich lipoproteins from the circulation. ${ }^{24,25)}$ In addition, apoE4 increases plasma LDL-C levels and the risk of atherosclerosis. ${ }^{23,26,27)}$ The increase in plasma LDL-C is caused by a down-regulated LDL receptor, when the enrichment of very low-density lipoprotein with apoE4 accelerates plasma cholesterol and lipoprotein clearance from the plasma by receptor-mediated endocytosis in the liver. ${ }^{28-30)}$ As mentioned above, plasma apoE levels are closely re-

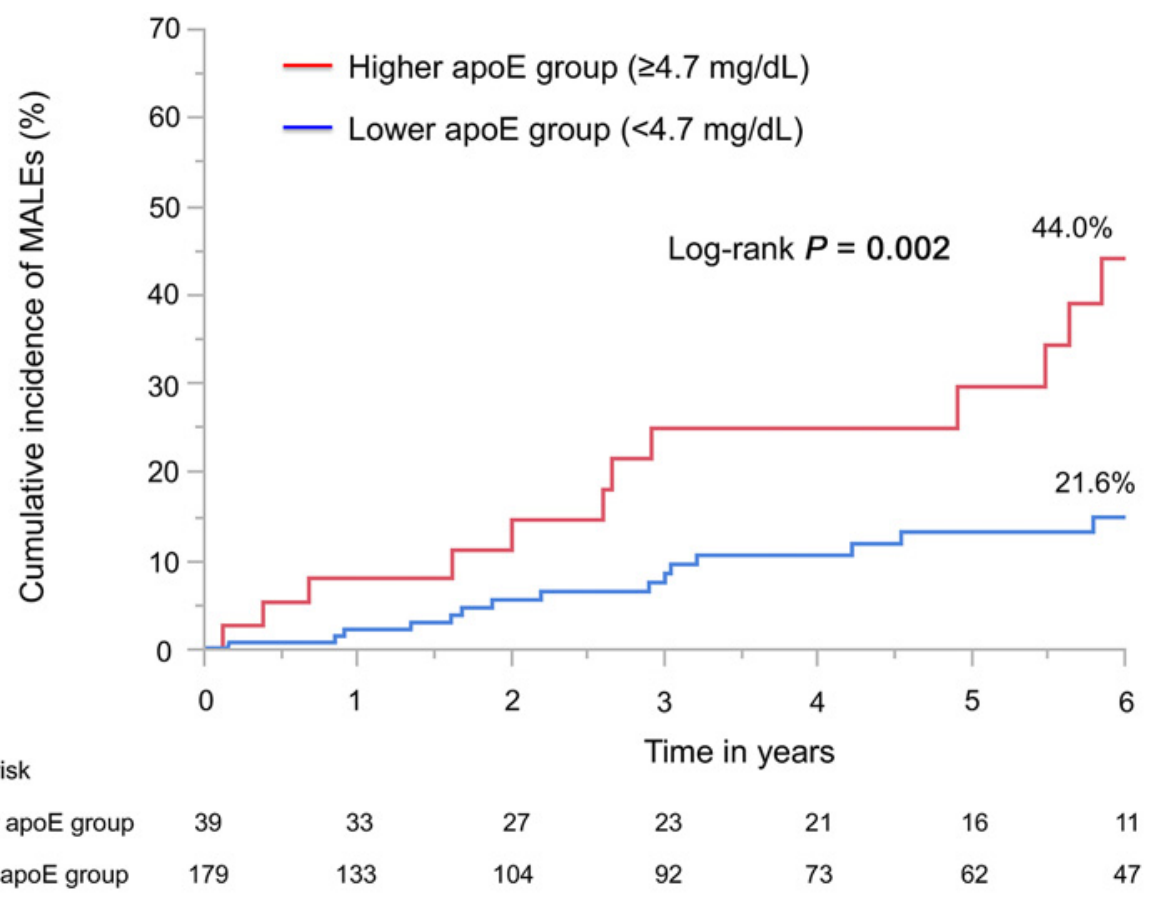

Figure 2. Kaplan-Meier curves for MALEs between the higher-apoE group and the lower-apoE group. MALEs composite endpoint defined as clinically driven target vessel revascularization and development of limb ischemia. ApoE indicates apolipoprotein E, and MALEs, major adverse limb events. 
Table III. Multivariable Cox Hazard Analysis of Major Adverse Limb Events

\begin{tabular}{lcccc}
\hline \multicolumn{1}{c}{ Covariate } & \multicolumn{2}{c}{ Univariate } & \multicolumn{2}{c}{ Multivariable } \\
& HR $(95 \% \mathrm{CI})$ & $P$-value & HR $(95 \% \mathrm{CI})$ & $P$-value \\
\hline Higher apoE level & $3.27(1.57-6.74)$ & 0.002 & $2.61(1.18-5.70)$ & 0.019 \\
TC, $10 \mathrm{mg}$ increase & $1.21(1.01-1.44)$ & 0.034 & $1.16(0.95-1.40)$ & 0.097 \\
TG, $10 \mathrm{mg} / \mathrm{dL}$ increase & $1.04(0.99-1.10)$ & 0.117 & & \\
Non-HDL-C, $10 \mathrm{mg} / \mathrm{dL}$ increase & $1.01(0.99-1.03)$ & 0.181 & & \\
Lower pre-treatment ABI & $1.64(0.77-3.47)$ & 0.195 & $1.93(0.89-4.19)$ & 0.141 \\
\hline
\end{tabular}

Higher apoE level was defined as apoE level $\geq 4.7 \mathrm{mg} / \mathrm{dL}$ (cutoff value); lower pre-treatment $\mathrm{ABI}$ was defined as pre-treatment $\mathrm{ABI}$ level $\geq 0.68$ (median value). ABI indicates the ankle-brachial index; ApoE, apolipoprotein E; HDL-C, high-density lipoprotein cholesterol; MALEs, major adverse limb events; TC, total cholesterol; and TG, triglyceride.

lated to serum triglyceride and serum LDL-C, which are more likely to have increased in the patients with PAD, thereby increasing the risk for exacerbation of ischemic symptoms in the lower limbs..$^{31-33)}$

Management of LDL-C levels is recommended in patients with PAD; however, consistent and clear reference values of LDL-C levels have not yet been defined in patients with intermittent claudication. The American College of Cardiology/American Heart Association guideline recommends statin treatment in all patients with intermittent claudication. However, no specific LDL-C value is shown (previously, it was recommended that the treatment goal was LDL-C $<100 \mathrm{mg} / \mathrm{dL}$ for all patients with PAD using statin). ${ }^{11)}$ In addition, the Trans-Atlantic InterSociety Consensus for the Management of PAD stipulated in 2007 that recommended goal LDL-C levels were $<100$ $\mathrm{mg} / \mathrm{dL}$ for all patients with PAD, and a more aggressive LDL-C was lowered to $<70 \mathrm{mg} / \mathrm{dL}$ for patients with atherosclerosis involving other vascular beds. ${ }^{18)}$ From the viewpoint of preventing cardiovascular events in patients with PAD, the treatment strategy of lowering LDL-C such as "the lower, the better" is certainly reasonable. ${ }^{34-37)}$ However, such strict treatment may be controversial regarding prevention of adverse limb events. Thus, for evaluating future adverse limb events and previous reports, we decided on LDL-C $<100 \mathrm{mg} / \mathrm{dL}$ as the treatment target for lowering LDL-C using statins. In this study, multivariable Cox hazard analysis revealed that apoE was the only strong independent predictor of MALEs in patients achieving LDL-C $<100 \mathrm{mg} / \mathrm{dL}$ on statin. The results of this study indicated a significant association between elevated apoE levels and residual risk for poor limb prognosis in patients with intermittent claudication undergoing statin treatment for lowering LDL-C. Furthermore, the higher-apoE group had significantly higher triglyceride levels and non-highdensity lipoprotein cholesterol levels. Thus, it is suggested that triglyceride therapy represented by fibrates may lead to reductions in future adverse limb events among individuals with intermittent claudication who have lower LDL-C levels and higher apoE levels.

This study had several limitations that require consideration. First, because of the single-center, retrospective, observational nature of the study, unknown confounding factors might have affected the outcomes regardless of analytical adjustments, and the relatively small number of enrolled patients limited the statistical power of the study. Second, the current study included only Japanese patients.
Thus, it is unclear whether the results can be applied to patients with intermittent claudication in other countries.

\section{Conclusion}

Higher preprocedural apoE levels at the time of the first intervention were significantly associated with adverse limb events in patients with intermittent claudication and lower LDL-C $<100 \mathrm{mg} / \mathrm{dL}$ on statin treatment. Thus, apoE levels could be a strong predictor for long-term limb prognosis and residual risk. Furthermore, to the best of our knowledge, this is the first report demonstrating the association between apoE and adverse limb events in patients with PAD; thus, the present findings warrant further investigation.

\section{Acknowledgments}

The authors are grateful to the staff of the Department of Cardiovascular Medicine at Juntendo University. The authors also appreciate the secretarial assistance of Ms. Yumi Nozawa.

\section{Disclosure}

Conflicts of interest: The authors declare that there are no conflicts of interest.

\section{References}

1. Anand SS, Bosch J, Eikelboom JW, et al. Rivaroxaban with or without aspirin in patients with stable peripheral or carotid artery disease: an international, randomised, double-blind, placebo-controlled trial. Lancet 2018; 391: 219-29.

2. Fakhry F, Spronk S, van der Laan L, et al. Endovascular revascularization and supervised exercise for peripheral artery disease and intermittent claudication: A randomized clinical trial. JAMA 2015; 314: 1936-44.

3. Hennrikus D, Joseph AM, Lando HA, et al. Effectiveness of a smoking cessation program for peripheral artery disease patients: a randomized controlled trial. J Am Coll Cardiol 2010; 56: $2105-12$.

4. Itoga NK, Tawfik DS, Lee CK, Maruyama S, Leeper NJ, Chang TI. Association of blood pressure measurements with peripheral artery disease events. Circulation 2018; 138: 1805-14.

5. Katsiki N, Giannoukas AD, Athyros VG, Mikhailidis DP. Lipidlowering treatment in peripheral artery disease. Curr Opin Pharmacol 2018; 39: 19-26. 
6. Suades R, Cosentino F, Badimon L. Glucose-lowering treatment in cardiovascular and peripheral artery disease. Curr Opin Pharmacol 2018; 39: 86-98.

7. Berger JS, Krantz MJ, Kittelson JM, Hiatt WR. Aspirin for the prevention of cardiovascular events in patients with peripheral artery disease: a meta-analysis of randomized trials. JAMA 2009; 301: 1909-19.

8. Heart Protection Study Collaborative Group. MRC/BHF Heart Protection Study of cholesterol lowering with simvastatin in 20,536 high-risk individuals: a randomised placebo-controlled trial. Lancet 2002; 360: 7-22.

9. Antoniou GA, Fisher RK, Georgiadis GS, Antoniou SA, Torella F. Statin therapy in lower limb peripheral arterial disease: systematic review and meta-analysis. Vascul Pharmacol 2014; 63: 79-87.

10. Giri J, McDermott MM, Greenland P, et al. Statin use and functional decline in patients with and without peripheral arterial disease. J Am Coll Cardiol 2006; 47: 998-1004.

11. Gerhard-Herman MD, Gornik HL, Barrett C, et al. 2016 AHA/ ACC guideline on the management of patients with lower extremity peripheral artery disease: A report of the American College of Cardiology/American Heart Association Task Force on Clinical Practice guidelines. Circulation 2017; 135: e726-79.

12. Mahley RW. Apolipoprotein E: from cardiovascular disease to neurodegenerative disorders. J Mol Med (Berl) 2016; 94: 73946.

13. Koopal C, Geerlings MI, Muller M, et al. The relation between apolipoprotein E (APOE) genotype and peripheral artery disease in patients at high risk for cardiovascular disease. Atherosclerosis 2016; 246: 187-92.

14. Ibánez B, James S, Agewall S, et al. 2017 ESC Guidelines for the management of acute myocardial infarction in patients presenting with ST-segment elevation. Rev Esp Cardiol (Engl Ed) 2017; 70: 1082 .

15. Collins R, Cranny G, Burch J, et al. A systematic review of duplex ultrasound, magnetic resonance angiography and computed tomography angiography for the diagnosis and assessment of symptomatic, lower limb peripheral arterial disease. Health Technol Assess 2007; 11: iii-iv, xi-xiii, 1-184.

16. Romano M, Mainenti PP, Imbriaco M, et al. Multidetector row $\mathrm{CT}$ angiography of the abdominal aorta and lower extremities in patients with peripheral arterial occlusive disease: diagnostic accuracy and interobserver agreement. Eur J Radiol 2004; 50: 303-8.

17. Menke J, Larsen J. Meta-analysis: accuracy of contrast-enhanced magnetic resonance angiography for assessing steno-occlusions in peripheral arterial disease. Ann Intern Med 2010; 153: 32534.

18. Norgren L, Hiatt WR, Dormandy JA, Nehler MR, Harris KA, Fowkes FGR. Inter-society consensus for the management of peripheral arterial disease (TASC II). J Vasc Surg 2007; 45 (Suppl S): S5-67.

19. Matsuo S, Imai E, Horio M, et al. Revised equations for estimated GFR from serum creatinine in Japan. Am J Kidney Dis 2009; 53: 982-92.

20. Huang YA, Zhou B, Wernig M, Südhof TC. ApoE2, ApoE3, and ApoE4 differentially stimulate APP transcription and $A \beta$ secretion. Cell 2017; 168: 427-41.e21.

21. Shore VG, Shore B. Heterogeneity of human plasma very low density lipoproteins. Separation of species differing in protein components. Biochemistry 1973; 12: 502-7.

22. Shelburne FA, Quarfordt SH. A new apoprotein of human plasma very low density lipoproteins. J Biol Chem 1974; 249: 1428-33.

23. Mahley RW, Weisgraber KH, Huang Y. Apolipoprotein E: structure determines function, from atherosclerosis to Alzheimer's disease to AIDS. J Lipid Res 2009; 50(Suppl): S183-8.

24. Utermann G, Kindermann I, Kaffarnik H, Steinmetz A. Apolipoprotein E phenotypes and hyperlipidemia. Hum Genet 1984; 65: 232-6.

25. Dallongeville J, Lussier-Cacan S, Davignon J. Modulation of plasma triglyceride levels by apoE phenotype: a meta-analysis. J Lipid Res 1992; 33: 447-54.

26. Mahley RW, Rall SC Jr. Apolipoprotein E: far more than a lipid transport protein. Annu Rev Genomics Hum Genet 2000; 1 : 507-37.

27. Bennet AM, Di Angelantonio E, Ye Z, et al. Association of apolipoprotein $\mathrm{E}$ genotypes with lipid levels and coronary risk. JAMA 2007; 298: 1300-11.

28. Gregg RE, Zech LA, Schaefer EJ, Stark D, Wilson D, Brewer $\mathrm{HB}$, Jr. Abnormal in vivo metabolism of apolipoprotein E4 in humans. J Clin Invest 1986; 78: 815-21.

29. Weintraub MS, Eisenberg S, Breslow JL. Dietary fat clearance in normal subjects is regulated by genetic variation in apolipoprotein E. J Clin Invest 1987; 80: 1571-7.

30. Li H, Dhanasekaran P, Alexander ET, Rader DJ, Phillips MC, Lund-Katz S. Molecular mechanisms responsible for the differential effects of apoE3 and apoE4 on plasma lipoproteincholesterol levels. Arterioscler Thromb Vasc Biol 2013; 33: 68793.

31. Ridker PM, Stampfer MJ, Rifai N. Novel risk factors for systemic atherosclerosis: a comparison of C-reactive protein, fibrinogen, homocysteine, lipoprotein(a), and standard cholesterol screening as predictors of peripheral arterial disease. JAMA 2001; 285: 2481-5.

32. Meade T, Zuhrie R, Cook C, Cooper J. Bezafibrate in men with lower extremity arterial disease: randomised controlled trial. BMJ 2002; 325: 1139.

33. Ness J, Aronow WS, Ahn C. Risk factors for symptomatic peripheral arterial disease in older persons in an academic hospital-based geriatrics practice. J Am Geriatr Soc 2000; 48: 312-4.

34. Cholesterol Treatment Trialists'(CTT) Collaboration, Baigent C, Blackwell L, et al. Efficacy and safety of more intensive lowering of LDL cholesterol: a meta-analysis of data from 170,000 participants in 26 randomised trials. Lancet 2010; 376: 1670-81.

35. Raymond C, Cho L, Rocco M, Hazen SL. New cholesterol guidelines: worth the wait? Cleve Clin J Med 2014; 81: 11-9.

36. Daida H, Teramoto T, Kitagawa Y, Matsushita Y, Sugihara M. The relationship between low-density lipoprotein cholesterol levels and the incidence of cardiovascular disease in high-risk patients treated with pravastatin: main results of the APPROACH-J study. Int Heart J 2014; 55: 39-47.

37. Boekholdt SM, Hovingh GK, Mora S, et al. Very low levels of atherogenic lipoproteins and the risk for cardiovascular events: a meta-analysis of statin trials. J Am Coll Cardiol 2014; 64: 48594.

\section{Supplemental Files}

Supplemental Figure

Please see supplemental files;

https://doi.org/10.1536/ihj.20-816 